\title{
Stationary Size Distributions of Growing Cells with Binary and Multiple Cell Division
}

\author{
M.M. Rading • T.A. Engel · R. Lipowsky • A. Valleriani
}

Received: 3 March 2011 / Accepted: 5 August 2011 / Published online: 10 September 2011

(C) The Author(s) 2011. This article is published with open access at Springerlink.com

\begin{abstract}
Populations of unicellular organisms that grow under constant environmental conditions are considered theoretically. The size distribution of these cells is calculated analytically, both for the usual process of binary division, in which one mother cell produces always two daughter cells, and for the more complex process of multiple division, in which one mother cell can produce $2^{n}$ daughter cells with $n=1,2,3, \ldots$ The latter mode of division is inspired by the unicellular algae Chlamydomonas reinhardtii. The uniform response of the whole population to different environmental conditions is encoded in the individual rates of growth and division of the cells. The analytical treatment of the problem is based on size-dependent rules for cell growth and stochastic transition processes for cell division. The comparison between binary and multiple division shows that these different division processes lead to qualitatively different results for the size distribution and the population growth rates.
\end{abstract}

Keywords Cell size distribution · Structured populations · Multiple cell division · Stochastic cell division · Population balance equations

\section{Introduction}

The study of proteins and metabolites in populations of unicellular organisms requires to sample a large number of cells from a culture and to extract their content for further analysis.

The research of M.M. Rading and T.A. Engel was supported by the BMBF in the framework of GoFORSYS.

M.M. Rading · T.A. Engel $\cdot$ R. Lipowsky $\cdot$ A. Valleriani $(\varangle)$

Department of Theory and Bio-Systems, Max Planck Institute of Colloids and Interfaces, Potsdam, Germany

e-mail: angelo.valleriani@mpikg.mpg.de

Present address:

T.A. Engel

Department of Neurobiology, Yale University School of Medicine, New Haven, CT, USA 


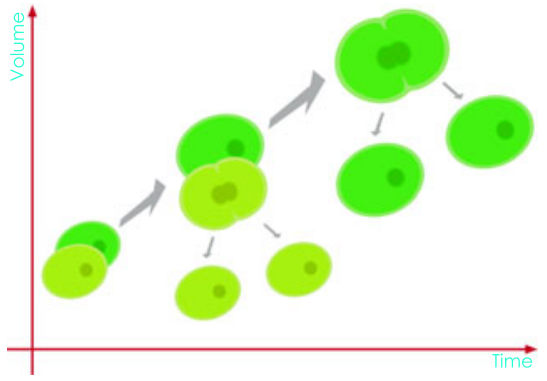

(a) Binary cell division

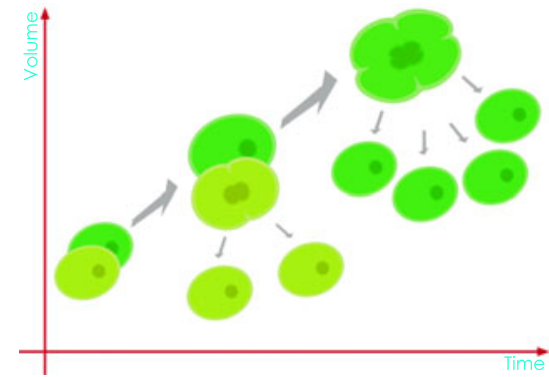

(b) Multiple cell division

Fig. 1 (Color online) Binary versus multiple cell division: (a) Cells of a unicellular species that undergoes only binary division. Two identical cells, one colored in light green, in the foreground, the other in dark green, in the background, start in the left bottom corner and grow until they divide. At a certain time, the light green cell in the foreground divides and produces two daughter cells while the dark green cell in the background continues to grow and to increase in volume. At a later time, the dark green cell in the background divides as well and produces two daughter cells, which are larger than the previously produced daughter cells; and (b) Cells of a unicellular species that can undergo multiple division. When the larger cell divides, it produces four daughter cells, which are not necessarily larger than the previously produced daughter cells

This procedure allows one to produce high-throughput data involving thousand of proteins and metabolites that are at the basis of the systems biology approach.

In order to obtain a sufficient amount of cell extract for subsequent analysis, it is necessary to sample over more than one million cells. If one assumes that the cells are all identical in their physiological state, sampling over such a large number of cells would provide a reliable average amount of all molecular components. However, we know from cell biology that cells can be in different phases of their cell cycle and that both gene expression and metabolism may be quite different in each of these phases [1]. Therefore, such a large sample of cells does not provide, in general, an average over cells that are in the same phase of the cell cycle, but rather over cells in many different phases.

Cell division in unicellular eukaryotes leads to the release of a certain number of daughter cells from a single mother cell. Although many species are known to produce only two daughter cells, there are species, most notably algae like Chlorella and in particular Chlamydomonas reinhardtii that produce a larger number of daughter cells depending on the size of the mother cell at the time of division [3-5]. Since the time and size of cell division are stochastic variables, sister cells will, in general, produce a varying number of daughter cells. Experiments with the unicellular organism Chlamydomonas reinhardtii in synchronized cultures [6] reveal that the timing and the division patterns depend on the growth conditions. On the other hand, the volume of the daughter cells at the time of release will strongly depend on the volume of the mother cell $[6,7]$. From experiments with single cells in micro chambers (on-chip culture measurement) $[9,10]$ we have a rough understanding of the growth processes and how growth trajectories depend on different phases of the cell cycle and light conditions. Thus, a relevant question concerns the stationary size distribution of cells under constant conditions such as those found in laboratory experiments. In particular, in order to allow a correct interpretation of the effect of parameter changes in the cell culture, it is necessary to know how the cell size distribution is affected by changes in the growth parameters. A schematic diagram illustrating the differences between binary and multiple cell division is shown in Fig. 1. 


\section{Theoretical Framework}

The mathematical analysis of cell cycles first arose from the observation of variable generation times in bacterial cells $[11,12]$. The random nature of the underlying processes required a reassessment of statistical properties such as generation times, age structure, and correlations between mother and daughter cells $[13,14]$. In order to address the fundamental structure of the mitotic cell cycle [15], stochastic models with distinct phases have been proposed, most notably by Smith and Martin [16]. In a different approach, the sequence of events that eventually leads to cell division is related to the cell cycle state, which is parametrized in terms of cell size.

In this latter approach, cell division is controlled by cell size, which determines the sequence of different events such as the initiation of DNA replication and the release of daughter cells. In fact, it is typically assumed that the molar concentrations of certain proteins and metabolites, as well as the rate of nutrients uptake and waste disposal are determined by the changing volume. Indeed, proteins and metabolites become to a certain extent diluted, as the volume grows and, both the uptake of nutrients and the disposal of waste depend on the cell surface, which grows as $v^{2 / 3}$ with volume $v$ for a roughly spherical cell shape.

A well known example for a model, in which the cell cycle is controlled by cell size, goes back to the work of Bell and Anderson [18], which can be cast in the more general framework of population balance equations (PBE) [19, 20]. The models specified therein quite generally allow for the balance of growth, division and partitioning mechanisms (see also Ref. [17]). A thorough presentation of key aspects of the mathematical framework is given by Ramkrishna in [20,21]. The stability and uniqueness of solutions of some simple models were systematically discussed in the literature [22-24]. However, because of the intricate functional form of the PBEs, it is difficult to find analytical solutions to these equations. Instead, numerical techniques for the analysis of PBEs have been developed that can be effectively implemented by Monte Carlo methods [21, 25, 26]. In the following analysis we apply these methods in order to study the process of multiple cell division.

Our study is motivated by the desire to understand the complex interactions that shape the size distribution of the unicellular algae Chlamydomonas reinhardtii, which we will abbreviate as 'chlamy'. This organism is one of the most studied model organisms in the research field of plant physiology [2]. Even though chlamy cells are cultivated in hundreds of laboratories around the globe, only a few attempts have been made to derive a suitable model for their cell cycle and stationary size distribution.

The typical cultivation conditions for chlamys correspond to a cell population, the density of which is kept constant by a continuous dilution of the growth medium. For these conditions, the number of cells grows exponentially. In non-synchronized populations, such as those kept under constant environmental conditions, each cell undergoes a period of growth and then divides. The special feature of chlamy is that the division of a mother cell can lead to $2^{n}$ daughter cells with $n=1,2,3, \ldots[6,7]$. This multiple division is quite different from the common binary division with $n=1$, and its effect on the size distribution of the cells is difficult to grasp intuitively. In fact, quite little is known about the mechanisms that regulate the division process and thus determine the number of offsprings.

One important target of the present study is to derive and solve a set of equations that can be used to compute the expected population growth (or proliferation) rate, and the size distribution of the cells under the simplifying assumption that the cell growth rate and the cell division rate depend only on the cell volume. The population growth rate is very important because it allows to assess the rate of dilution of the cultivation in order to keep a constant density and constant quality of the growth medium. 
Another objective of our study is to elucidate the response of cells to changes in the external growth conditions. In order to develop our theoretical description, it is useful to summarize some experimental observations. Experiments on synchronized chlamy cells revealed that the volume of each cell after birth grows in proportion to the area of the cell surface [9]. Another set of experiments on such cells also shows that cells committed to cell division typically divide as often as they can, provided that the volume of the daughter cells exceeds a minimal volume [8]. This latter observation indicates that the cell volume at the time of division provides a constraint on the maximum number of cell divisions. ${ }^{1}$ Previous studies on other unicellular organisms undergoing binary cell division have shown that the rate of cell division is fairly constant [16]. For such populations, the rate of cell division is thus related to the population growth rate or to the so-called population half time. In contrast, in the present study, we will consider unicellular organisms that undergo multiple cell division and take the dependence of the cell division rate on the cell volume into account.

\section{The General Equation}

Let each cell in the culture at time $t$ be characterized by its age $\tau$ and its current volume $v$. We shall also assume, in agreement with the experimental observation, that there is a minimum volume $v_{0}$ below which cells are not viable. Thus, throughout the whole manuscript we shall assume that there is a volume $v_{0}>0$ such that all cell volumes $v$ satisfy the inequality $v \geq v_{0}$. We will hereafter assume that the growth conditions in the culture are constant, independent of $t$. The generic law that governs the volume growth in time is then expressed as

$$
\frac{\mathrm{d} v}{\mathrm{~d} t}=f(v, \tau),
$$

for $v \geq v_{0}$, where $f(v, \tau)$ is the volume- and age-dependent growth rate of a single cell. We have mentioned in the introduction that under certain conditions $f$ turns out to be proportional to the cell surface and independent on $\tau$ but we will keep its more general form for the time being.

The division rate of each cell is described by the function $p(v, \tau)$, which gives the probability $p(v, \tau) \delta t$ that a cell of size $v$ and age $\tau$ divides in the next small interval of time $\delta t$. The existence of a minimum size $v_{0}$ constrains also the rate $p$ in such a way that $p(v, \tau)=0$ for $v \leq 2 v_{0}$. The latter condition will be implicitly assumed throughout manuscript. As we shall see later, an additional condition on $p(v)$ is that it attains a positive and constant value for large $v$. On the other hand, we do not need to impose any constraint on the maximal volume of the cells.

Once the two basic functions $f(v, \tau)$ and $p(v, \tau)$ have been defined, the number $N$ of cells at time $t$ with volume in $[v, v+\mathrm{d} v]$ and age in $[\tau, \tau+\mathrm{d} \tau]$ is given by $N(v, \tau, t) \mathrm{d} v \mathrm{~d} \tau$. The function $N$ obeys the partial differential equation (PDE) $[18,20]$

$$
\partial_{t} N+\partial_{\tau} N+\partial_{v}(f(v, \tau) N)+(D+p(v, \tau)) N=0,
$$

for $v \geq v_{0}$, with $\partial_{x}$ denoting the partial derivative with respect to the variable $x$ and $D$ being a uniform dilution rate, which reflects the experimental procedure of replacing a fraction of

\footnotetext{
${ }^{1}$ Detailed observations have shown that the start of cell division and the release of the daughter cells is separated by a short, intermediate time period, during which the daughter cells are kept within the cell wall of the mother cell and, thus, do not affect the cell volume statistics of free cells. In the present study, we will ignore this short intermediate time period.
} 
the cell culture by fresh medium. A derivation of (2) is given in Appendix A. This equation holds for $\tau>0$ whereas for age $\tau=0$ the number $N$ represents the number of newborn cells. Thus, one of the advantages of using both age and volume is that the creation of new cells via cell division enters as a boundary condition for $\tau=0$ in (2). A similar approach, aimed at following the population over time, one generation after the other, was also proposed in [27]. In the case studied in Ref. [27], the boundary conditions for $\tau=0$ have been imposed for each generation.

Since we are interested in the size and age distribution and not in the number of cells, we can then define the density $\Phi$ via

$$
N(v, \tau, t) \equiv n(t) \Phi(v, \tau, t)
$$

where now $n(t)$ is the number of cells at time $t$. Plugging (3) into (2) leads then to

$$
\partial_{t} \Phi+\partial_{\tau} \Phi+\partial_{v}(f(v, \tau) \Phi)+\left(\partial_{t} \log n+D+p(v, \tau)\right) \Phi=0
$$

for $v \geq v_{0}$. Under the typical experimental conditions, the population size is growing exponentially as $n(t) \sim \exp [(\gamma-D) t]$. In this case, we have that $\partial_{t} \log n=\gamma-D$ is a constant and if $p$ and $f$ do not depend on $t$, we can therefore look for the stationary solution

$$
\Phi^{\mathrm{st}}(v, \tau)=\lim _{t \rightarrow \infty} \Phi(v, \tau, t)
$$

which satisfies the simpler PDE

$$
\partial_{\tau} \Phi^{\mathrm{st}}+\partial_{v}\left(f(v, \tau) \Phi^{\mathrm{st}}\right)+(\gamma+p(v, \tau)) \Phi^{\mathrm{st}}=0
$$

for $v \geq v_{0}$, where $\gamma$, the constant population growth rate, replaces the previous term $\partial_{t} \log n$ and the dilution rate $D$ is canceled out. Adding the boundary conditions

$$
\Phi^{\mathrm{st}}(v, 0)=B(v),
$$

which will be discussed in detail later, (6) is the standard advection equation with variable coefficients that can be solved with the method of characteristic functions (see Ref. [29] and Appendix B). Even though we could write down the general solution of (6) this solution would provide only relatively little insights in the processes considered here. As we shall see in the next section it is useful to first simplify some terms in the equation. In addition, we shall see in the next section how the division process completely determines the boundary conditions (7). The dependence of $f$ and of $p$ on the age $\tau$ reflects the intuitive expectation that certain processes may be age dependent. The growth rate $f$, for instance, may depend on the specific cell cycle phases, and the transition from one phase to the next may depend on the completion of certain sub-processes. Likewise, cell division may depend on the completion of a series of check-points that require some time.

Experimental results for the age dependence of the cell growth rate function $f$ and the cell division rate $p$ are still lacking. Therefore, we will make the simplifying assumption that $f(v, \tau)$ and $p(v, \tau)$ do not depend on $\tau$. Under these conditions, we can rewrite (6) as

$$
\partial_{\tau} \Phi^{\mathrm{st}}+\partial_{v}\left(f(v) \Phi^{\mathrm{st}}\right)+(\gamma+p(v)) \Phi^{\mathrm{st}}=0,
$$

for $v \geq v_{0}$ with the boundary conditions (7). Using

$$
\Gamma(v)=\gamma+\mathrm{d} f(v) / \mathrm{d} v+p(v)
$$


this equation can be solved by standard methods (see Appendix B). The result is

$$
\phi(v)=\int_{v_{0}}^{v} \mathrm{~d} z \frac{B(z)}{f(z)} \exp \left(-\int_{z}^{v} \mathrm{~d} y \frac{\Gamma(y)}{f(y)}\right),
$$

where

$$
\phi(v) \equiv \int_{0}^{\infty} \mathrm{d} \tau \Phi^{\mathrm{st}}(v, \tau)
$$

is the marginal probability density which depends on the boundary function $B(v)$ that we are going to specify in detail below. It is important to stress that the right hand side of (9) is completely determined by the choice of $f(v)$ and $p(v)$ (and thus of $v_{0}$ ). The value of the population growth rate $\gamma$ is not an independent variable and is implicitly determined by $B(v)$ and by the normalization of (9).

\section{Binary Division}

The boundary condition (7) determines $\Phi^{\text {st }}(v, 0)$, i.e., the size distribution of newborn cells having age zero. We will assume symmetric cell division, meaning that the volume of each mother cell is equally partitioned among its daughter cells. Therefore, the boundary condition should relate the size of newborn cells to the size of their mother cells. This relation is simply given if one keeps in mind that to generate a newborn cell with volume in the range $[v, v+\mathrm{d} v]$ it is necessary that the mother cell had volume in $[2 v, 2 v+\mathrm{d}(2 v)]$. At this point, it is clear that, for $v \geq v_{0}$,

$$
\Phi^{\mathrm{st}}(v, 0) \mathrm{d} v=2 \int_{0}^{\infty} \mathrm{d} \tau p(2 v, \tau) \Phi^{\mathrm{st}}(2 v, \tau) \mathrm{d}(2 v)
$$

where the 2 in front of the integral is related to the fact that binary division produces two identical cells. Thus, after eliminating $\mathrm{d} v$ from both sides of the equation, we have the boundary condition

$$
\Phi^{\mathrm{st}}(v, 0)=4 \int_{0}^{\infty} \mathrm{d} \tau p(2 v, \tau) \Phi^{\mathrm{st}}(2 v, \tau) .
$$

It now follows from (7) that the rhs of (11) determines $B(z)$ in (9). If we specialize again to the case, in which the division rate $p$ is independent of age $\tau$, we finally have

$$
B(z)=4 p(2 z) \phi(2 z)
$$

so that the solution (9) for binary division becomes

$$
\phi(v)=\exp \left(-\int_{v_{0}}^{v} \mathrm{~d} y \frac{\Gamma(y)}{f(y)}\right) \cdot \int_{v_{0}}^{v} \mathrm{~d} z \frac{4 p(2 z) \phi(2 z)}{f(z)} \exp \left(\int_{v_{0}}^{z} \mathrm{~d} y \frac{\Gamma(y)}{f(y)}\right),
$$

which represents an implicit relation for $\phi(v)$ since this function appears on both sides of the equation. After the substitution

$$
\phi(v) \equiv r(v) \exp \left(-\int_{v_{0}}^{v} \mathrm{~d} y \frac{\Gamma(y)}{f(y)}\right)
$$


which defines the new function $r(v)$, we finally obtain the implicit equation

$$
r(v)=\int_{v_{0}}^{v} \mathrm{~d} z K(z) r(2 z)
$$

with the kernel

$$
K(v)=\frac{4 p(2 v)}{f(v)} \exp \left(-\int_{v}^{2 v} \mathrm{~d} y \frac{\Gamma(y)}{f(y)}\right),
$$

which we assume to be smooth for $v$ around $v_{0}$ and to decrease to zero sufficiently fast for large $v$. One should notice that the relation (15) is a direct consequence of the fact that the density $\phi(v)$ appears on both sides of (13). For $v=v_{0}$, we have that $r\left(v_{0}\right)=0$ while for large $v$ the function $r(v)$ reaches the asymptotic positive value

$$
\lim _{v \rightarrow \infty} r(v)=r_{\infty}
$$

Using this asymptotic value in (15), we obtain

$$
r(v)=r_{\infty}-\int_{v}^{\infty} \mathrm{d} z K(z) r(2 z),
$$

so that the solution can be obtained in an iterative manner, which leads to

$$
r(v)=r_{\infty}\left(1-\int_{v}^{\infty} \mathrm{d} z_{1} K\left(z_{1}\right)+\int_{v}^{\infty} \mathrm{d} z_{1} K\left(z_{1}\right) \int_{2 z_{1}}^{\infty} \mathrm{d} z_{2} K\left(z_{2}\right)+\cdots\right)
$$

or to

$$
r(v)=\lim _{M \rightarrow \infty} r_{\infty}\left(1+\sum_{m=1}^{M}(-1)^{m} \int_{v}^{\infty} \mathrm{d} z_{1} K\left(z_{1}\right) \cdots \int_{2 z_{m-1}}^{\infty} \mathrm{d} z_{m} K\left(z_{m}\right)\right),
$$

for $v \geq v_{0}$. Once (20) is inserted into (14), the probability density is determined up to a multiplicative constant $r_{\infty}$, which can be fixed by normalization. It is then clear that in the limit of large $v$ the probability density behaves as

$$
\phi(v) \rightarrow r_{\infty} \exp \left(-\int_{v_{0}}^{v} \mathrm{~d} y \frac{\Gamma(y)}{f(y)}\right),
$$

a result that is consistent with the analysis of symmetric cell division as performed in [28]. In the same work, it was shown that this asymptotic behavior occurs also under generic assumptions about asymmetric division. We can re-write (20) as

$$
r(v)=r_{\infty} R(v)
$$

with $R(v) \rightarrow 1$ for large $v$. Once we insert $r(v)$ in (15), we will find that (15) is satisfied if and only if $R\left(v_{0}\right)=0$, namely if and only if

$$
R\left(v_{0}\right) \equiv \lim _{M \rightarrow \infty}\left(1+\sum_{m=1}^{M}(-1)^{m} \int_{v_{0}}^{\infty} \mathrm{d} z_{1} K\left(z_{1}\right) \cdots \int_{2 z_{m-1}}^{\infty} \mathrm{d} z_{m} K\left(z_{m}\right)\right)=0,
$$


with $K(v)$ defined in (16). Since, in general, $R\left(v_{0}\right)$ will depend on the value of $\gamma$, the condition (23) can be used to determine the value of the population growth factor $\gamma$. In general this condition may be satisfied by more than one value of the parameter $\gamma$ so that an additional condition is necessary in order to select the correct one. Indeed, once the distribution $\phi(v)$ is determined one can also find the equation that determines the value of the population growth rate

$$
\gamma=\int_{2 v_{0}}^{\infty} \mathrm{d} v p(v) \phi(v),
$$

which takes into account that for any two newborn cells there is one mother cell less and that division can occur only for a cell volume larger or equal to $2 v_{0}$. This equation contains the unknown $\gamma$ on both sides, in the rhs through the function $\Gamma(y)$ and can be solved in general only numerically.

\subsection{Explicit Solution for a Particular Choice of Parameters}

As an application of our theoretical solution, we consider here the case in which we set $v_{0}=0, f=1 \mu \mathrm{m}^{3} / \mathrm{h}$ and $p=1 \mathrm{~h}^{-1}$. This simple case reminds the growth law of the bacterium $E$. coli and the choice of the rates guarantees that $\gamma=1$, as can be easily verified from (24). Equation (16) gives

$$
K(v)=4 \exp [-(\gamma+1) v]
$$

which, once inserted in (20) gives

$$
R(v)=1-\frac{4}{\gamma+1} \sum_{m=0}^{\infty}(-1)^{m}\left(\frac{4}{\gamma+1}\right)^{m} \frac{\exp \left[-(\gamma+1) \sigma_{m} v\right]}{\pi_{m}},
$$

where $\sigma_{m}=\sum_{j=0}^{m} 2^{j}$ and $\pi_{m}=\prod_{j=0}^{m} \sigma_{j}$. This solution shows that $R(v) \rightarrow 1$ when $v \rightarrow$ $\infty$, in agreement with (18). On the other hand, one has also to find the value of $\gamma$ that satisfies (23). The numerical estimation of (23) with (26) as function of $\gamma$ shows that the only physical value that verifies $R(0)=0$ is given by $\gamma=1 \mathrm{~h}^{-1}$. By inserting $R(v)$ thus obtained in (14) finally gives the volume distribution

$$
\phi(v)=r_{\infty} R(v) \exp (-(\gamma+1) v)
$$

which, by means of the normalization, provides a value of $r_{\infty} \sim 6.9 \mathrm{~h}^{-1}$. It is not difficult to verify both analytically and numerically (see Fig. 2 ) that (27) provides the correct solution for the given choice of the parameters $f$ and $p$.

\section{Multiple Division}

When considering the process that leads to multiple division, we should take into special consideration the fact that mother cells can divide up to a maximum number of identical daughter cells. This maximum number is determined by the fact that daughter cells cannot be smaller than a certain minimal viable volume $v_{0}$.

To find out how the size of the mother cell determines the number of cell divisions, we should consider the following fact. If a mother cell at the commitment time has volume in $\left[2 v_{0}, 4 v_{0}\right)$ then it can divide only one time and produce only two daughter cells. If, instead, a mother cell at the commitment time has volume in $\left[4 v_{0}, 8 v_{0}\right)$ it can divide up to two 


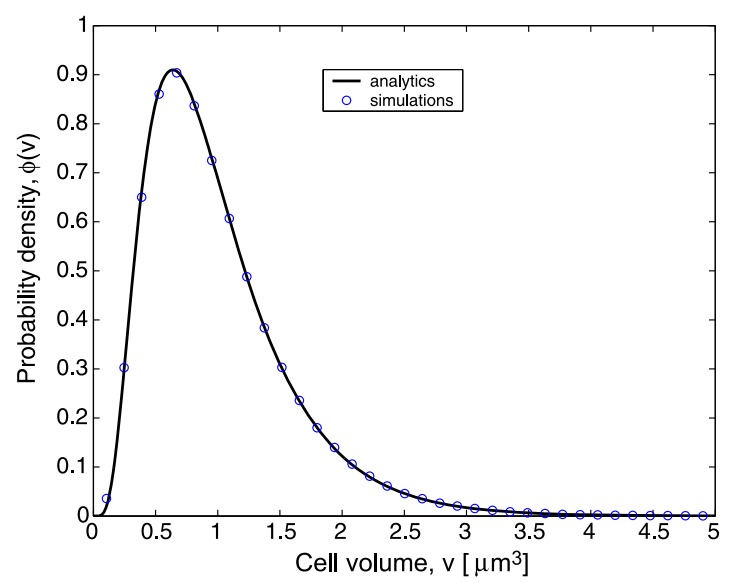

Fig. 2 (Color online) Probability density $\phi$ as function of cell size $v$ : Comparison of analytical results for the marginal probability density $\phi$, defined in (9a) and computed explicitly in (27), and stochastic simulations for the simple division rule with $v_{0}=0$ and $f=1 \mu \mathrm{m}^{3} / \mathrm{h}$ and $p=1 \mathrm{~h}^{-1}$. The particularly good agreement between analytical results and simulations shows that the calculation based on the expansion (26) is correct. The analytical result is obtained by using (26) truncated after the first 20 terms. The number of terms included in the truncation was determined by the condition that the inclusion of additional terms would hardly improve the numerical accuracy. The stochastic simulations have been performed on a fixed population of $N=10^{3}$ cells for $10^{6}$ generations after stabilization of the probability density

times and produce a maximum of four daughter cells. Generalizing this pattern, we see that a mother cell that has a volume in $\left[2^{k} v_{0}, 2^{k+1} v_{0}\right.$ ) can divide up to $k$ times and produce a maximum of $2^{k}$ daughter cells, for $k \geq 1$.

Let us thus denote with $k \geq 1$ the maximal number of divisions for a given mother cell. It is therefore possible to think that, conditional on $k$, this mother cells may choose to divide a number $1 \leq i \leq k$ of times according to a probability mass $M_{i \mid k}$. Quite generally, for $k \geq 1, M_{i \mid k}$ obeys a normalization condition $\sum_{i=1}^{k} M_{i \mid k}=1$, meaning that cells divide with certainty. Therefore, $M_{1 \mid k}=1$ simply describes a binary division process while $M_{k \mid k}=1$ describes a division process in which the cell produces the maximum amount of daughter cells. Thus, daughter cells with volume $v \in\left[2^{j} v_{0}, 2^{j+1} v_{0}\right)$ can be produced from the division of mother cells in size $\left[2^{k} v_{0}, 2^{k+1} v_{0}\right.$ ) dividing $k-j$ times according to the probability $M_{k-j \mid k}$ for any $k \geq j+1$. To put it formally, let us define the intervals

$$
I_{j}=\left[2^{j} v_{0}, 2^{j+1} v_{0}\right)
$$

for $j=0,1,2, \ldots$ and the index function $\Theta_{j}(v)$ defined as

$$
\Theta_{j}(v)= \begin{cases}1 & \text { if } v \in I_{j}, \\ 0 & \text { otherwise }\end{cases}
$$

If we now define the stationary density as

$$
\Phi_{j}^{\mathrm{st}}(v, \tau)=\Phi^{\mathrm{st}}(v, \tau) \Theta_{j}(v)
$$

with the obvious property that $\Phi=\sum_{j} \Phi_{j}$, this division process leads to the boundary condition 


$$
\Phi_{j}^{\mathrm{st}}(v, 0) \mathrm{d} v=\sum_{k=j+1}^{\infty} M_{k-j \mid k} 2^{k-j} \int_{0}^{\infty} \mathrm{d} \tau p\left(2^{k} v, \tau\right) \Phi^{\mathrm{st}}\left(2^{k} v, \tau\right) \mathrm{d}\left(2^{k} v\right) .
$$

Finally, the boundary condition for the distribution $\Phi$ is given by

$$
\Phi^{\mathrm{st}}(v, 0)=\sum_{j=0}^{\infty} \Phi_{j}^{\mathrm{st}}(v, 0)
$$

for any $v \geq v_{0}$. If we force all cells to undergo a binary division process by imposing $M_{1 \mid k}=$ 1 , one can easily see that (32) leads to (10). On the other hand, if we impose the maximal division through $M_{k \mid k}=1$, then only $j=0$ contributes in (31) and (32) becomes

$$
\Phi^{\mathrm{st}}(v, 0) \mathrm{d} v=\sum_{k=1}^{\infty} 2^{k} \int_{0}^{\infty} \mathrm{d} \tau p\left(2^{k} v, \tau\right) \Phi\left(2^{k} v, \tau\right) \mathrm{d}\left(2^{k} v\right)
$$

for $v_{0} \leq v<2 v_{0}$, while for any value of $v$ outside this interval we have $\Phi^{\text {st }}(v, 0)=0$.

In the following, we will restrict the analysis to the case in which a mother cell always divides to the maximum number of daughter cells. This assumption is supported by the experimental observations made in Refs. [6-8]. We will namely impose that $M_{k \mid k}=1$. If we assume now a division rate $p(v)$ that depends only on the volume and not on age, the reasoning proceeds in analogy to the case with simple binary division and leads to a boundary function given by

$$
B(v)=\Phi^{\mathrm{st}}(v, 0)=\sum_{k=1}^{\infty} 2^{2 k} p\left(2^{k} v\right) \phi\left(2^{k} v\right)
$$

which is strictly valid only for $v_{0} \leq v<2 v_{0}$ and is zero otherwise. Plugging this into the solution (9) we have

$$
\phi(v)=\exp \left(-\int_{v_{0}}^{v} \mathrm{~d} y \frac{\Gamma(y)}{f(y)}\right) \cdot \sum_{k=1}^{\infty} 4^{k} \int_{v_{0}}^{\min \left(2 v_{0}, v\right)} \mathrm{d} z \frac{p\left(2^{k} z\right) \phi\left(2^{k} z\right)}{f(z)} \exp \left(\int_{v_{0}}^{z} \mathrm{~d} y \frac{\Gamma(y)}{f(y)}\right),
$$

where $\Gamma(v)=\gamma+\mathrm{d} f(v) / \mathrm{d} v+p(v)$. Equation (35) shows that the behavior of $\phi(v)$ for $v>2 v_{0}$ is determined solely by the first exponential term. This is also intuitive because in this range of volume there are no contributions coming from newborn cells. Using this fact, we can conclude that for $v>2 v_{0}$ the density behaves as

$$
\phi(v)=r_{\infty} \exp \left(-\int_{v_{0}}^{v} \mathrm{~d} y \frac{\Gamma(y)}{f(y)}\right) .
$$

Since the argument of the function $\phi$ inside the integral (35) is always larger than $2 v_{0}$ we can insert (36) in the integral and obtain

$$
\phi(v)=r_{\infty} \exp \left(-\int_{v_{0}}^{v} \mathrm{~d} y \frac{\Gamma(y)}{f(y)}\right) \sum_{k=1}^{\infty} \int_{v_{0}}^{\min \left(2 v_{0}, v\right)} \mathrm{d} z K(z, k),
$$

where we have defined the kernel

$$
K(z, k)=4^{k} \frac{p\left(2^{k} z\right)}{f(z)} \exp \left(-\int_{z}^{2^{k} z} \mathrm{~d} y \frac{\Gamma(y)}{f(y)}\right) .
$$


Inserting now (37) for $v>2 v_{0}$ and equating it with the asymptotic form in (36), we can find out that the kernel satisfies

$$
\sum_{k=1}^{\infty} \int_{v_{0}}^{2 v_{0}} \mathrm{~d} z K(z, k)=1
$$

which, in general, cannot be satisfied by any arbitrary choice of the functions $f(v)$ and $p(v)$. The condition (39) therefore determines the only parameter left free, namely the population growth rate $\gamma$. On the other hand, once $\phi(v)$ is known, it is possible to derive the population growth rate $\gamma$ also using

$$
\gamma=\sum_{k=1}^{\infty}\left(2^{k}-1\right) \int_{2^{k} v_{0}}^{2^{k+1} v_{0}} \mathrm{~d} z p(z) \phi(z)=\sum_{k=1}^{\infty}\left(2^{k}-1\right) 2^{k} \int_{v_{0}}^{2 v_{0}} \mathrm{~d} z p\left(2^{k} z\right) \phi\left(2^{k} z\right),
$$

where the coefficients in front of the integrals come from the fact that for every $2^{k}$ new (daughter) cells there is one mother cell less in the whole population. One should notice that (40) determines $\gamma$ implicitly, since this parameter appears on both sides of the equation through the function $\Gamma(y)$. The integral on the rhs for any fixed $k$ is proportional to the number of cells that divide $k$ times. This observation allows us to define the fraction $q_{k}$ of dividing cells that undergo $k$ divisions as

$$
q_{k}=\frac{\int_{2^{k} v_{0}}^{2^{k+1} v_{0}} \mathrm{~d} z p(z) \phi(z)}{\int_{2 v_{0}}^{\infty} \mathrm{d} z p(z) \phi(z)}=2^{k-1} \frac{\int_{v_{0}}^{2 v_{0}} \mathrm{~d} z p\left(2^{k} z\right) \phi\left(2^{k} z\right)}{\int_{v_{0}}^{\infty} \mathrm{d} z p(2 z) \phi(2 z)},
$$

for $k \geq 1$. Depending on the choice of $p(v)$ and the growth law $f(v)$ we can expect to see varying division patterns with a dominant frequency $q_{k}$ of reproduction with $2^{k}$ daughter cells.

\subsection{A Toy Model Calculation}

We would like now to verify that (39) is a condition that provides the population growth rate $\gamma$. We can do this by considering a toy model in which we set $p(v)=\theta\left(v-2 v_{0}\right) \mathrm{h}^{-1}$ and $f=1 \mu \mathrm{m}^{3} / \mathrm{h}$. After some algebra it is possible to verify that (39) is given by

$$
\sum_{k=1}^{\infty} 4^{k} \frac{\exp \left[-\left(a_{k}-2\right) v_{0}\right]}{a_{k}}\left(1-\exp \left[-a_{k} v_{0}\right]\right)=1
$$

where $a_{k}=2^{k}(\gamma+1)-\gamma$. Given that all other parameters are fixed, this equation can be satisfied by certain values of $\gamma$, which will obviously depend on the applied value of $v_{0}$. If one then numerically evaluates (42) as function of $\gamma$ one can find the zeros of this equation. We find: for $v_{0}=1 \mu \mathrm{m}^{3}$ we get $\gamma \sim 0.48 \mathrm{~h}^{-1}$ while for $v_{0}=2 \mu \mathrm{m}^{3}$ we have $\gamma \sim 0.28 \mathrm{~h}^{-1}$, and for $v_{0}=3 \mu \mathrm{m}^{3}$ we have $\gamma \sim 0.20 \mathrm{~h}^{-1}$.

To verify this result, we can first make the following simple reasoning. The cells will divide after an average time equal to $1 / p$ after that they have reached the size $2 v_{0}$. The average size at division will then be given by $2 v_{0}+f / p$, which for our choice of the parameters is equivalent to $2 v_{0}+1$, irrespective of the initial size of the cells. It is easy to verify that for $v_{0}>0.5$ the division process allows only a binary division and thus the average size of newborn cells will be given by $v_{0}+1 / 2$. We will now assume that the average new born cells 


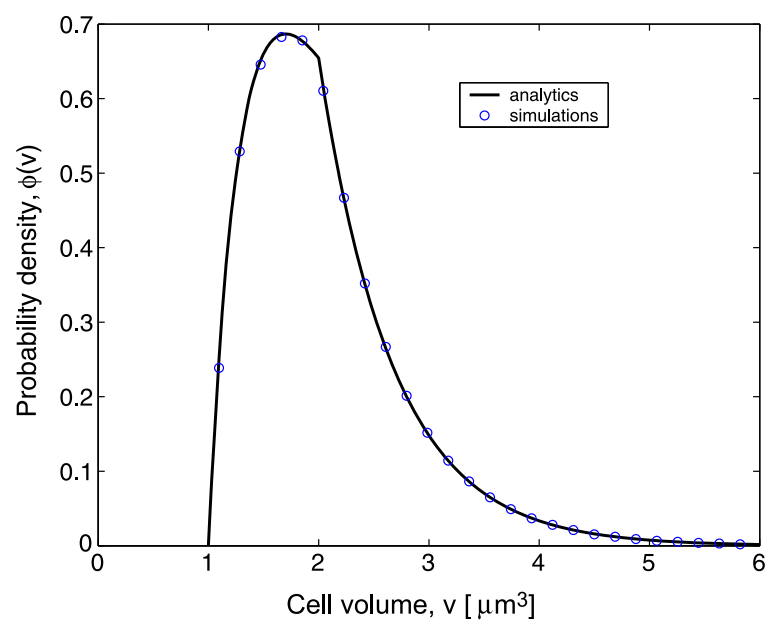

Fig. 3 (Color online) Probability density $\phi$ as function of cell size $v$ : Comparison of analytical results from (45) and stochastic simulations for the multiple division rule with $v_{0}=1 \mu^{3}$ and $f=1 \mu \mathrm{m}^{3} / \mathrm{h}$ and $p(v)=\theta\left(v-2 v_{0}\right) \mathrm{h}^{-1}$. The particularly good agreement between analytical results and simulations shows

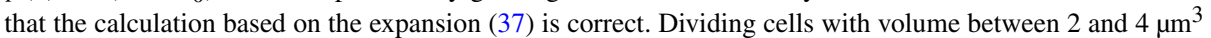
produce only two daughter cells, while dividing cells with $v \geq 4$ would produce more daughter cells. The analytical result has been obtained by truncating the sum in (35) after the first 20 terms. The number of terms included in the truncation was determined by the condition that the inclusion of additional terms would hardly improve the numerical accuracy. The simulations have been performed starting from a population of $N=10^{3}$ cells and taking $10^{3}$ sample histograms every $10^{4}$ cell divisions

are representative for the whole cell population. Thus, the time required to double the size of the population is given by the time required by the average new born cell to get divided. This time is given by $\Delta t=v_{0}+1 / 2$. Since we know that this is the time required to double the population, we can now estimate $\gamma$ as follows

$$
\gamma \Delta t \sim \log \left(\frac{N(t+\Delta t)}{N(t)}\right)=\log 2,
$$

which leads to

$$
\gamma \sim \frac{\log 2}{v_{0}+0.5}
$$

which gives $\gamma \sim 0.46,0.28,0.20$ for $v_{0}=1,2,3 \mu \mathrm{m}^{3}$, respectively. As we can see, the agreement with the computation from (42) is excellent. In addition to that, it is possible to write the explicit form of the probability density $\phi(v)$ once one takes into account that its behavior is different for $v \in\left[v_{0}, 2 v_{0}\right.$ [ and for $v \geq 2 v_{0}$. Indeed, we have

$$
\begin{gathered}
\phi\left(v_{0} \leq v<2 v_{0}\right)=r_{\infty} \exp ^{-\gamma\left(v-v_{0}\right)} \sum_{k=1}^{\infty} \frac{4^{k}}{a_{k}} \exp \left(2 v_{0}\right)\left(\exp \left(-a_{k} v_{0}\right)-\exp \left(-a_{k} v\right)\right) \\
\phi\left(v \geq v_{0}\right)=r_{\infty} \exp \left(-\gamma v_{0}\right) \exp \left(-(\gamma+1)\left(v-2 v_{0}\right)\right),
\end{gathered}
$$

where $a_{k}=2^{k}(\gamma+1)-\gamma$ and the constant $r_{\infty}$ can be fixed by normalizing the density. The match between the analytical solution and stochastic simulations is shown in Fig. 3. 


\section{Solutions for Empirically Relevant Forms of the Growth and Division Rates}

Depending on growth conditions, the cultured cells grow and divide differently. In order to analyze the impact of various growth conditions on the sampling statistics during balanced growth one needs to know the approximate functional form that describes growth and transition to cell division.

\subsection{Growth Rates}

Here we will concentrate on special cases with empirical relevance for chlamy. From onchip measurements with the organism we gain the knowledge of the functional form of the growth law, which is explicitly size dependent [9]. It has to be taken into consideration that the growth rate of the cell volume depends on the surface of the cell. This would immediately lead to the assumption that the function $f(v)$ used in $(1)$ is given by

$$
f(v)=f_{0} v^{2 / 3},
$$

where $f_{0}$ has dimensions of length divided by time and is thus proportional to the growth velocity of the cell diameter. The model (46) does not include erratic and noisy growth conditions or varying growth properties in cells. In this work, we will assume that the growth velocity $f_{0}$ is some deterministic parameter depending solely on the growth conditions and we will leave a discussion about its possible variations to further studies. Of course increasing transport ways within the cell and energy consumption of growing cells would counter balance this purely surface related growth and we could easily add, at least in first order, terms of the form $-f_{1} v$ to (46). For green algae, however, (46) is an appropriate model and indicates linear growth in cell diameter at least in cell cultures under experimental conditions. Thus a rapid increase in cell volume is observed which in turn is corroborated by a large productivity evidently observed in organisms like Chlorella and chlamy.

\subsection{Cell Division Rate}

Cells need a minimal volume $v_{0}$ in order to be viable and to be able to grow and reproduce. It is reasonable to assume that the cell division rate, namely the rate by which a cell commits itself to start division, is zero if this cell has a volume smaller than a critical size $v_{c}$. From an experimental point of view one could determine the transition rate in cohort experiments. In these experiments, one would monitor a set of cells that start from identical physiological states just after release and grow under fixed conditions. Tracking then each single cell over its life cycle would provide the statistics of the dividing cells with respect to size and age. This statistics is condensed in the survival function $\Psi_{d}(v)$, which is the probability density function of dividing cells having size $v$. With the help of $\Psi_{d}(v)$, one can determine the desired division rate $p(v)[27]$ according to

$$
p(v)=\frac{\Psi_{d}(v) f(v)}{1-\int^{v} \Psi_{d}(u) \mathrm{d} u},
$$

which depends solely on the unknown distribution $\Psi_{d}(v)$ and where $f(v)$ is the growth rate. The rate $p(v)$ defined in (47) is sometimes referred to as the hazard rate [30].

From the theoretical point of view, a meaningful transition rate $p(v)$ should always be strictly positive for large $v$. As a limiting behavior, it should at least satisfy

$$
\lim _{v \rightarrow \infty} p(v)=C>0
$$


where $C$ is an arbitrary constant. This condition is also known as the condition of strictly increasing hazard rate (IHR). The condition (48) now allows to determine the limiting form of the function $\Psi_{d}(v)$ at large $v$. Indeed, taking into account that both numerator and denominator of (47) tend to zero as $v \rightarrow \infty$, in this limit we obtain

$$
p(v) \rightarrow-f(v) \frac{\mathrm{d}}{\mathrm{d} v}\left[\log \left(f(v) \Psi_{d}(v)\right)\right]
$$

which, once inserted in (48) gives

$$
\Psi_{d}(v) \approx \frac{1}{f(v)} \exp \left(-\int^{v} \frac{1}{f(u)} \mathrm{d} u\right) .
$$

This limiting form of $\Psi_{d}(v)$ tells us that any function that decreases less rapidly than (50) is not a good candidate as a survival function because it does not satisfy (48). One such function is, for example, the log-normal distribution.

Since we do not know the form of the survival function $\Psi_{d}(v)$, we will henceforth assume that the condition (48) holds from any volume $v \geq v_{c}$. In particular, we will set $v_{c}=2 v_{0}$ in order to ensure that cells to be committed to cell division produce viable cells. Thus, the simplest functional form for $p(v)$ that satisfies these conditions is given by

$$
p(v)=\beta \Theta\left(v-2 v_{0}\right),
$$

where $\Theta$ is the Heaviside function and $v_{c}=2 v_{0}$ holds for analytical convenience.

\subsection{Detailed Analysis}

Here, we would like to determine the size distribution under the assumptions (46) and (51) both for the binary and the multiple division process. The sensitivity of these two cases to changing growth conditions $f_{0}$ is one particular focus of the analysis.

For binary division, it is not difficult but laborious to verify that one can find an expression in terms of an infinite sum of exponential functions for the function $R(v)$, which was defined in (22). It is also possible to see, by means of numerical verification, that the expansion in (20) converges very quickly so that already the first ten terms are more than sufficient to characterize the function.

The same considerations hold also for the expansion concerning the multiple division of cells as it was derived in (37), (38), and (39) for the exact derivation of the population growth rate. In fact, the computation of the analytical expression for these variables is somewhat simpler than in the case of the binary cell division. Still, the explicit formulation of these functions would not increase our understanding of the phenomena.

For these reasons, we will present our analysis by including results from both stochastic simulations of the population dynamics and numerical integration of the equations. The plots of the relevant quantities are shown in Figs. 4(a) and 4(b) while a simplified analysis is given in Appendix C. From quite general experimental observations [7] in chlamy cultures the minimum cell size has been estimated to be $v_{0} \approx 20 \mu \mathrm{m}^{3}$ and as a rough estimate for the transition rate we choose $\beta=1 / 8 \mathrm{~h}^{-1}$. The value of $\beta$ is fixed and independent of the growth velocity $f_{0}$. Figure 4 compares the impact of different growth velocities $f_{0}$ on the size spectra for binary and multiple division but otherwise unchanged division rules. Figures 4(a) and 4(b) show how $f_{0}$ affects the heterogeneity of cells during a balanced growth phase for a multiple division process. One can see that a doubling of the growth velocity has 


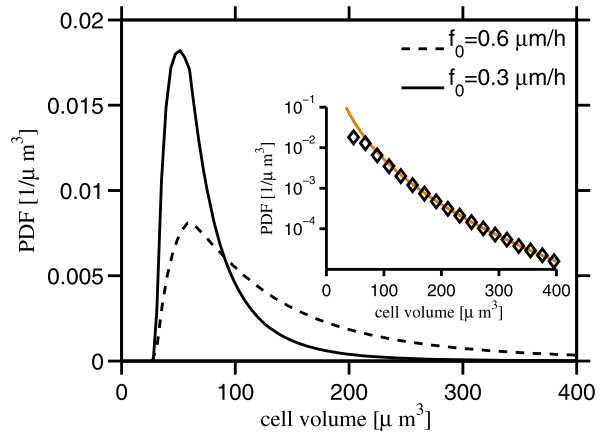

(a) Binary cell division

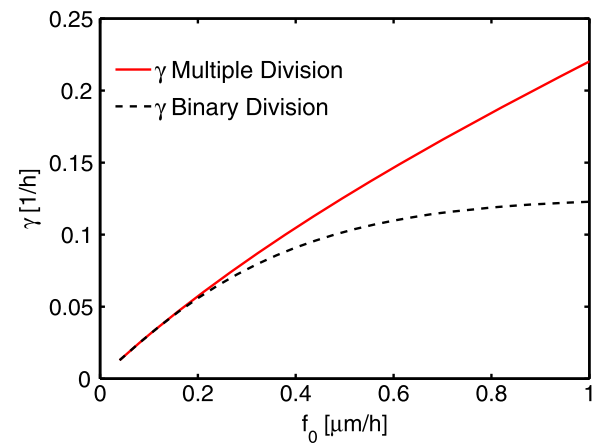

(c) Population growth rate

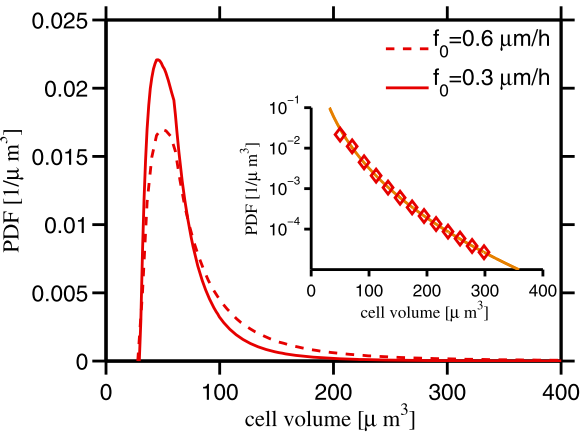

(b) Multiple cell division

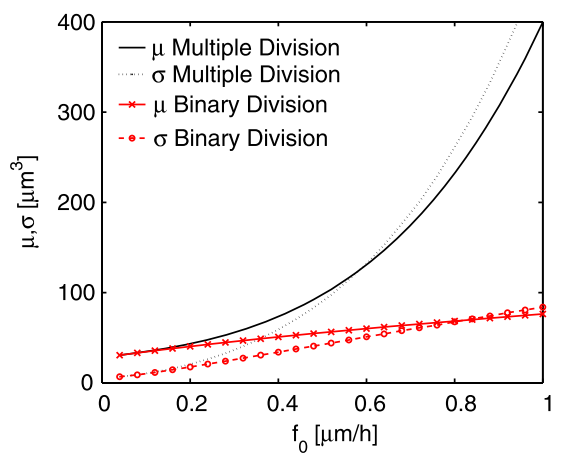

(d) Average cell size and variance

Fig. 4 (Color online) Probability density function (PDF) of the cell volume $v$ and population growth rate for binary and multiple cell division. Plots (a) and (b) show the different ways in which the cell volume shifts to larger cell sizes in both cases of multiple and binary division for two choices of the growth velocities $\left(f_{0}=0.3 \mu \mathrm{m} / \mathrm{h}\right.$ and $\left.f_{0}=0.6 \mu \mathrm{m} / \mathrm{h}\right)$, respectively. In the insets, we compare the results from stochastic simulations of the population dynamics (diamonds) with the asymptotic behavior given by (21) and (36), respectively, with $f_{0}=0.3 \mu \mathrm{m} / \mathrm{h}$. Plot (c): Dependence of the population growth rate $\gamma$ on the growth velocity $f_{0}$. Plot (d) compares the average cell size and standard deviation of the size distribution for both division processes as function of the growth velocity $f_{0}$ for $\beta=0.125 \mathrm{~h}^{-1}$. A typical value of $f_{0}$ in experiments is $0.5 \mu \mathrm{m} / \mathrm{h}$. Inspection of (d) reveals that the standard deviation $\sigma$ grows with $f_{0}$ for both division processes and that this growth is more pronounced for binary division

a much larger impact on the size distribution for binary division than on the size distribution for the multiple division. This is due to the fact that in the latter case larger cells arising from a larger value of $f_{0}$ simply produce more daughter cells, which all have to have an initial size between $v_{0}$ and $2 v_{0}$. In the case of binary division instead, the larger value of $f_{0}$ produces larger mother cells, which in turn produce larger daughter cells. This concept is illustrated also in the cartoon shown in Fig. 1. Using the implicit formulas given in (24) and (40) for the population growth rate $\gamma$ we find the dependence on $f_{0}$. In any case, $\gamma$ is a strictly increasing function of $f_{0}$ but the difference between binary and multiple cell division appears for larger growth velocities $f_{0}$ depending on $v_{0}$ (see Fig. 4(c)). Whereas the population growth rate scales roughly linearly in the multiple division scenario, it is instead bounded by some maximum value $\gamma_{\max }=\beta$ in the case of strictly binary division. Setting the transition rate $\beta$ also defines the parameter range of $f_{0}$ for which both division types start to diverge. Figure 4(d) clearly demonstrates a shift in the size structure for both types of division. Here, the structure of the population is given in terms of the average cell size $\mu$ and the standard deviation $\sigma$ representing the width of the spectrum. In the relevant range of growth velocities $f_{0}$ 
we expect minor changes in the case of multiple division, whereas binary division illustrates the opposite extreme of huge variations within the population structure after relatively small variations of growth conditions.

Thus, at the level of size distributions, we see that both the average cell volume and the standard deviation associated to the distribution grow with increasing $f_{0}$. In particular, we expect that with binary division the whole distribution moves to larger values with a mean value that grows roughly like $f_{0}^{3}$, see (70). With multiple division instead, we expect to see a distribution clearly more peaked at values between $v_{0}$ and $2 v_{0}$ and a longer and longer tail for larger and larger values of $f_{0}$. This difference could not be more dramatical. Indeed, with binary division the whole population responds to a change of the environmental conditions, as long as this change is encoded in $f_{0}$. With multiple division instead, larger and larger values of $f_{0}$ induce the coexistence of an ever increasing number of many small cells with few very large ones.

\section{Conclusions}

During balanced growth a culture of unicellular organisms adapts to specific growth conditions and approaches a stationary phase. This assertion also holds for division and growth cycles with high complexity. The interplay between growth and division constrains the population structure of physiological properties, such as the cell size. The more detailed analysis is often restricted to models that capture just a few aspects of the cell cycle, although quite intricate and interdependent mechanisms do exist. The underlying events are often subtle and produce durable inter-generational correlations, asynchrony of growth and division cycles, and heterogeneities concerning both the cell specific growth pattern and the number of cells released. Aside from most of these complex aspects, our main objective was to elucidate the differences between a common binary division scheme and multiple cell division at the level of cell size distribution. In particular, our parameter choice and the knowledge of the experimental aspects are both focused on cell cultures of Chlamydomonas reinhardtii. We have assumed, for the multiple cell division, a cascading process of cell fission which coincidentally describes the most productive division pattern. Applying this simplified sketch of multiple cell division we are able to link growth properties and division rules to population growth and to the frequency of divisions into a given number of daughter cells and we have been able to find analytical expressions that take into account very general forms of the functions related to the growth and division rates. Though potentially more realistic, we have left complicated rules out of consideration and restrict the analysis to its simplest functional form. By doing this we observe a quite sensitive difference between binary and multiple division rules as functions of energy supply, parameterized by the growth velocity $f_{0}$. We have observed that the multiple division rounds alter the physiological structure of the population with unsaturated population growth rates. As a consequence, we have seen, on one hand, that with multiple division most of the population remains confined in the same range of volume while a few individuals tend to be very large in volume. On the other hand, with binary division the whole population responds in a rather homogeneous way to an increase in the growth velocity but still the differences in volume, as measured by the standard deviation of the stationary distribution, grow also quite considerably.

As mentioned above, the theory developed in this manuscript can include any functional form of the cell growth and cell division rates as long as these forms lead to a stationary cell size distribution, as is usually the case in the experimental set-ups. The choices for these functions made in our computations are reasonable simple assumptions but we hope 
that this work may inspire some experiments both with steady state and synchronized cell culture to determine the nature of these functions more precisely and thus test our model.

Acknowledgements The authors would like to thank M. Steup, J. Ruprecht, A. Nagar, O. Ebenhöh, M. Sandmann, H. Brust, H. Lockstein, B. Müller-Röber, S. Klumpp and R. Marathe for useful discussions. The illustration in Fig. 1 was designed in collaboration with Susann Valleriani.

Open Access This article is distributed under the terms of the Creative Commons Attribution Noncommercial License which permits any noncommercial use, distribution, and reproduction in any medium, provided the original author(s) and source are credited.

\section{Appendix A: Derivation of the Main PDE}

Let $N(v, \tau, t)$ the function that gives the number of cells in $[v, v+\mathrm{d} v]$ and $[\tau, \tau+\mathrm{d} \tau]$. For $\tau>0$ and for any $v_{1}$ and $v_{2}$ such that $v_{0}<v_{1}<v_{2}<\infty$ we have

$$
\begin{aligned}
& \int_{v_{1}}^{v_{2}} \mathrm{~d} v N(v, \tau+\mathrm{d} \tau, t+\mathrm{d} t) \\
& =\int_{v_{1}}^{v_{2}} \mathrm{~d} v N(v, \tau, t)[1-p(v, \tau) \mathrm{d} t][1-D \mathrm{~d} t] \\
& \quad+\int_{v_{1}-f\left(v_{1}, \tau\right) \mathrm{d} t}^{v_{1}} \mathrm{~d} v N(v, \tau, t)-\int_{v_{2}-f\left(v_{2}, \tau\right) \mathrm{d} t}^{v_{2}} \mathrm{~d} v N(v, \tau, t),
\end{aligned}
$$

which, taking only the first order terms in $\mathrm{d} t$, leads to (2).

Indeed, the first term on the right is given by division and dilution while the last two terms are given by growing into the interval $\left[v_{1}, v_{2}\right)$ and cells growing out of the interval, respectively. Expanding all terms and keeping only the first-order terms in $\mathrm{d} t$ and $\mathrm{d} \tau$ gives

$$
\begin{aligned}
& \int_{v_{1}}^{v_{2}} \mathrm{~d} v\left(N(v, \tau, t)+\partial_{\tau} N(v, \tau, t) \mathrm{d} \tau+\partial_{t} N(v, \tau, t) \mathrm{d} t\right) \\
& =\int_{v_{1}}^{v_{2}} \mathrm{~d} v[N(v, \tau, t)(1-p(v, \tau) \mathrm{d} t-D \mathrm{~d} t)] \\
& \quad-\left(N\left(v_{2}, \tau, t\right) f\left(v_{2}, \tau\right) \mathrm{d} t-N\left(v_{1}, \tau, t\right) f\left(v_{1}, \tau\right) \mathrm{d} t\right),
\end{aligned}
$$

where $\partial_{x}$ is the partial derivative with respect to $x$. Taking now into account that the last term can be rewritten as

$$
\left(N\left(v_{2}, \tau, t\right) f\left(v_{2}, \tau\right)-N\left(v_{1}, \tau, t\right) f\left(v_{1}, \tau\right)\right)=\int_{v_{1}}^{v_{2}} \mathrm{~d} v \frac{\partial}{\partial v}(N(v, \tau, t) f(v, \tau, t)),
$$

and putting all terms into one integral, one obtains

$$
\int_{v_{1}}^{v_{2}} \mathrm{~d} v\left[\left(\partial_{t}+\partial_{\tau}+p(v, \tau)+D\right) N(v, \tau, t)+\partial_{v}(N(v, \tau, t) f(v, \tau))\right]=0 .
$$

Since this equality holds for any arbitrary choice of $v_{1}$ and $v_{2}$ that satisfy $v_{0}<v_{1}<v_{2}<\infty$, the integrand must be also zero in this interval and this leads to the differential equation (2). One can notice that the process of cell division does not enter into the derivation of the equation. In fact, cell division creates new cells having age $\tau=0$ and thus appears as a boundary condition for $N(v, 0, t)$. The particular form of the boundary condition will thus entirely depend on the form of the division process. 


\section{Appendix B: Solution of the PDE}

We start with (8)

$$
\partial_{\tau} \Phi^{\mathrm{st}}+\partial_{v}\left(f(v) \Phi^{\mathrm{st}}\right)+(\gamma+p(v)) \Phi^{\mathrm{st}}=0,
$$

for $v \geq v_{0}$ with generic boundary conditions (7). The solution of this equation now proceeds as follows. Let us consider two variables $k_{0}$ and $s$ that parameterize the trajectories on the $(v, \tau)$ plane according to the following laws

$$
\frac{\mathrm{d} \tau}{\mathrm{d} s}=1 \quad \text { and } \quad \frac{\mathrm{d} v}{\mathrm{~d} s}=f(v),
$$

and $k_{0}=v(s=0)$. Since now we have that $\tau \geq 0$ it turns out that $s=\tau$ and therefore it holds that

$$
\int_{k_{0}}^{v} \mathrm{~d} x \frac{1}{f(x)}=\tau
$$

and so

$$
I(v)-I\left(k_{0}\right)=\tau
$$

if $I(v)$ is the primitive function of $f(v)^{-1}$. The expression (59) provides an implicit dependence between the parameters of the characteristic curve

$$
\begin{gathered}
v(s)=I^{-1}\left(I\left(k_{0}\right)+s\right) \\
k_{0}=I^{-1}(I(v)-\tau) .
\end{gathered}
$$

By applying now the parameterization given in (57) we find out that $\Phi^{\text {st }}$ obeys the ordinary differential equation (ODE)

$$
\frac{\mathrm{d} \Phi^{\mathrm{st}}(s)}{\mathrm{d} s}+\Gamma(s) \Phi^{\mathrm{st}}(s)=0
$$

where

$$
\Gamma(v)=\gamma+\frac{\mathrm{d} f(v)}{\mathrm{d} v}+p(v),
$$

with boundary conditions $\Phi^{\text {st }}(s=0)=B\left(k_{0}\right)$.

The solution of the ODE leads to

$$
\Phi^{\mathrm{st}}(v(s), \tau(s))=B\left(k_{0}\right) \exp \left(-\int_{0}^{s} \mathrm{~d} x \Gamma(v(x))\right),
$$

so that fixing now $v$ and $\tau$ we can substitute the back transformations (60) and we obtain

$$
\Phi^{\mathrm{st}}(v, \tau)=B\left(I^{-1}(I(v)-\tau)\right) \exp \left(-\int_{0}^{\tau} \mathrm{d} x \Gamma\left(I^{-1}\left(I\left(k_{0}\right)+x\right)\right)\right),
$$

and using now again (59) for $v$ and $\tau$ fixed, we have

$$
\Phi^{\mathrm{st}}(v, \tau)=B\left(I^{-1}(I(v)-\tau)\right) \exp \left(-\int_{0}^{\tau} \mathrm{d} x \Gamma\left(I^{-1}(I(v)-\tau+x)\right)\right) .
$$


We obtain a considerable simplification if we consider now the marginal probability density $\phi(v)$ defined as

$$
\phi(v)=\int_{0}^{\infty} \mathrm{d} \tau \Phi^{\mathrm{st}}(v, \tau) .
$$

In fact, integrating both sides of (65) in $\tau$ leads to

$$
\phi(v)=\int_{0}^{\infty} \mathrm{d} \tau B\left(I^{-1}(I(v)-\tau)\right) \exp \left(-\int_{0}^{\tau} \mathrm{d} x \Gamma\left(I^{-1}(I(v)-\tau+x)\right)\right),
$$

and transforming now $z=I^{-1}(I(v)-\tau)$ for fixed $v$ we have

$$
\phi(v)=\int_{v_{0}}^{v} \mathrm{~d} z \frac{B(z)}{f(z)} \exp \left(-\int_{0}^{I(v)-I(z)} \mathrm{d} x \Gamma\left(I^{-1}(I(z)+x)\right)\right),
$$

and changing again the variable in $y=I^{-1}(I(z)+x)$ we finally have (9)

$$
\phi(v)=\int_{v_{0}}^{v} \mathrm{~d} z \frac{B(z)}{f(z)} \exp \left(-\int_{z}^{v} \mathrm{~d} y \frac{\Gamma(y)}{f(y)}\right)
$$

which still includes the boundary function $B(v)$.

\section{Appendix C: Simplified Analysis of the Empirically Relevant Case}

Let us start with the binary division and let us observe that the typical size $v_{m}$ of a mother cell at the moment of division is independent of the initial size. In fact, $v_{m}$ depends only on the time elapsed after having passed the threshold $2 v_{0}$ for cell division. We shall estimate this time with $1 / \beta$. It is then possible to find out that the typical size at cell division is then given by

$$
v_{m}=\left(\frac{f_{0}}{3 \beta}+\left(2 v_{0}\right)^{1 / 3}\right)^{3},
$$

which leads, according to the binary division process, to an initial size $v_{d}$ of a daughter cell given by $v_{d}=v_{m} / 2$. One first conclusion, from this calculation, is that the typical size of a random cell would roughly be in between $v_{m} / 2$ and $v_{m}$ and that, therefore, this typical size would grow monotonously with increasing $f_{0}$.

To estimate the population growth rate, we shall find out what is the typical time $\Delta t$ until a cell divides starting from the initial size of a daughter cell. This typical time will depend on the volume $v_{d}$. In fact, if $v_{d}<2 v_{0}$ the typical time $\Delta t$ to division will be given by $\Delta t=\tau+1 / \beta$, where $\tau$ is the time required to reach the volume $2 v_{0}$ starting from the volume $v_{d}$. On the other hand, if $v_{d}>2 v_{0}$ then we have simply $\Delta t=1 / \beta$. Thus, it is important to find out for which values of $f_{0}$ we have $v_{d}<2 v_{0}$. It turns out that this holds if $f_{0}<\bar{f}_{0}$ where

$$
\bar{f}_{0}=3 \beta\left(2 v_{0}\right)^{1 / 3}\left(2^{1 / 3}-1\right),
$$

which leads to an estimate of $\tau$ given by

$$
\tau=\frac{\overline{f_{0}}-f_{0}}{2^{1 / 3} \beta f_{0}}
$$


for $f_{0} \leq \bar{f}_{0}$ and $\tau=0$ otherwise.

If we assume now that the whole cell population divides in a synchronous way a time $\Delta t$ after birth, we have that the whole population doubles ${ }^{2}$ after exactly $\Delta t$ units of time thus giving

$$
N(t+\Delta t)=2 N(t),
$$

where $\Delta t$ is given by $\tau+1 / \beta$. Considering that, in this approximation, $N(t+\Delta t)=$ $N(t) \exp (\gamma \Delta t)$ we have the following estimates for $\gamma$

$$
\gamma \sim \begin{cases}\frac{2^{1 / 3} f_{0}}{2^{1 / 3} f_{0}+\left(\bar{f}_{0}-f_{0}\right)} \beta & \text { for } f_{0}<\bar{f}_{0}, \\ \beta & \text { for } f_{0} \geq \bar{f}_{0}\end{cases}
$$

where we have used the fact that asymptotically for large $f_{0}$ it must result that $\gamma=\beta$. We see from this computation that the population growth rate $\gamma$ increases as a function of $f_{0}$ for $f_{0}<\bar{f}_{0}$ and then it remains constant for larger values of $f_{0}$. Let us remind that $f_{0}$ is a proxy for the amount of nutrients or energy provided to the cells. The behavior found here indicates that in the binary cell division an increase in $f_{0}$ leads to an increase of the cell volumes and less to an increase of the number of cells.

The situation for multiple cell division is now much simpler. Since the time of cell division does not depend on the mechanism of division and is determined by the rate $p(v)=\beta \Theta\left(v-2 v_{0}\right)$, the volume $v_{m}$ of mother cells at the time of division is the same as the one computed before and it is given by (70). On the other hand, the maximum number $k$ of cell divisions is given by

$$
k=\operatorname{int}\left(\frac{\log \left(v_{m} / v_{0}\right)}{\log 2}\right)
$$

where the function $\operatorname{int}(x)$ takes the integer part of the real number $x$, so that the daughter cells have initial volume $v_{d}=v_{m} / 2^{k}$ which is always in $v_{0} \leq v_{d}<2 v_{0}$.

With $\tau \sim 1 / f_{0}$, we see that the time $\Delta t$ until division is changing little with $f_{0}$, as it was also the case in the binary division. On the other hand, the number of daughter cells depends on $f_{0}$ through $k$ given in (75). Thus, for $\Delta t=a / f_{0}+1 / \beta$, where $a$ is some constant, we have

$$
N(t+\Delta t) \sim 2^{k} N(t)
$$

with $k$ given in (75), which leads to $\gamma \sim \beta k$. For large $f_{0}$ and taking into account $v_{m}$ given in (70) this leads then to

$$
\gamma \sim \beta \log \left(f_{0} / \beta\right)
$$

which is then increasing with $f_{0}$. Due to the large number of daughter cells, instead, the mean cell volume will finally tend to some limit roughly given by

$$
\langle v\rangle \sim v_{d}+v_{m} / 2^{k},
$$

\footnotetext{
${ }^{2}$ In reality, the estimation that we are making here is not completely correct. In fact, for $f_{0} \gg \bar{f}_{0}$ the system would be well described by a pure birth process. After a time $\Delta t=1 / \beta$ the average population size would increase by a factor $e$ and not by a factor 2 .
} 
where $k$ is given in (75) and has thus a light growth as a function of $f_{0}$. The conclusion is therefore that in the multiple division case an increase in the growth factor $f_{0}$ leads primarily to a growth of the population size and less or little to a growth of the mean cell volume.

\section{References}

1. Albert, B., Johnson, A., Lewis, J., Raff, M., Roberts, K., Walter, P.: Molecular Biology of the Cell. Garland Science Textbooks (2007)

2. Harris, E.H.: Chlamydomonas as a model organism. Annu. Rev. Cell Biol. Plant Mol. Biol. 52, 363-406 (2001)

3. Spudich, J.L., Sager, R.: Regulation of the Chlamydomonas cell cycle by dark and light. J. Cell Biol. 85, 136-145 (1980)

4. Donnan, L., John, P.C.L.: Cell cycle control by timer and sizer in Chlamydomonas. Nature 304, 630-633 (1983)

5. Craigie, R.A., Cavaliere-Smith, T.: Cell volume and the control of the Chlamydomonas cell cycle. J. Cell Sci. 54, 173-191 (1982)

6. Oldenhof, H., Zachleder, V., van den Ende, H.: Cell cycle of Chlamydomonas $r$. Folia Microbiol. 53, 52 (2007)

7. Oldenhof, H., Zachleder, V., van den Ende, H.: Blue and red light regulation in the cell cycle of Chlamydomonas reinhardtii. Eur. J. Phycol. 41, 313-320 (2006)

8. Zachleder, V., van den Ende, H.: Cell cycle events in the green alga Chlamydomonas eugametos and their control by environmental factors. J. Cell Sci. 102, 469-474 (1992)

9. Matsumura, K., Yagi, T., Yasuda, K.: Role of timer and sizer in regulation of Chlamydomonas cell cycle. Biochem. Biophys. Res. Commun. 306, 1042-1049 (2003)

10. Wakamoto, Y., Inoue, I., Moriguchi, H., Yasuda, K.: Analysis of single-cell differences by use of an on-chip microculture system and optical trapping. Fresenius J. Anal. Chem. 371, 276-281 (2001)

11. Rahn, O.: A chemical explanation of the variability of the growth rate. J. Gen. Physiol. 15, 257-277 (1932)

12. Kendall, D.G.: On the choice of a mathematical model to represent normal bacterial growth. Biometrika 35, $316(1948)$

13. Powell, E.O.: Growth rate and generation time of bacteria, with special reference to continuous cultures. J. Gen. Microbiol. 15, 492-511 (1956)

14. Fantes, P.A., Nurse, P.: Division timing: controls, models and mechanisms. Cell Cycle 1, 11-33 (1981)

15. Karp, G.: Cellular reproduction. In: Cell and Molecular Biology, pp. 570-612. Wiley, New York (2008) (Chap. 14)

16. Smith, J.A., Martin, L.: Do cells cycle? Proc. Natl. Acad. Sci. USA 70, 1263-1267 (1973)

17. Brenner, N., Shokef, Y.: Nonequilibrium statistical mechanics of dividing cell populations. Phys. Rev. Lett. 99, 138102 (2007)

18. Bell, G.I., Anderson, E.C.: Cell growth and division I. A mathematical model with applications to cell volume distributions in mammalian suspension cultures. Biophys. J. 7, 329-351 (1967)

19. Subramanian, G., Ramkrishna, D., Fredrickson, A.G., Tsuchiya, H.M.: On the mass distribution model for microbial cell populations. Bull. Math. Biophys. 32, 521-537 (1970)

20. Ramkrishna, D., Borwanker, J.D.: A puristic analysis of population balance I. Chem. Eng. Sci. 28, 14231435 (1973)

21. Ramkrishna, D.: Population Balances. Academic Press, San Diego (2005)

22. Diekmann, O., Heijmans, H.J.A.M., Thieme, H.R.: On the stability of the cell size distribution. J. Math. Biol. 19, 227-248 (1984)

23. Heijmans, H.J.A.M.: On the stable size distribution of populations reproducing by fission into two equally parts. Math. Biosci. 72, 19-50 (1984)

24. Tyson, J.J., Hannsgen, K.B.: The distribution of cell size and generation time in a model of the cell cycle incorporating size control and random transitions. J. Theor. Biol. 113, 29-62 (1985)

25. Mantzaris, N.V., Liou, J., Daoutidis, P., Srienc, F.: Numerical solution of a mass structured cell population balance model in an environment of changing substrate concentration. J. Biotechnol. 71, 157-174 (1999)

26. Shah, B.H., Borwanker, J.D., Ramkrishna, D.: Monte Carlo simulation of microbial population growth. Math. Biosci. 31, 1-23 (1976)

27. Liou, J., Srienc, F., Fredrickson, A.G.: Solutions of population balance models based on a successive generation approach. Chem. Eng. Sci. 92, 1529-1540 (1996) 
28. Friedlander, T., Brenner, N.: Cellular properties and population asymptotics in the population balance equation. Phys. Rev. Lett. 101, 018104 (2008)

29. Debnath, L.: Nonlinear Partial Differential Equations. Birkhäuser, Basel (2007)

30. Beichelt, F., Montgomery, D.C.: Teubner-Taschenbuch der Stochastik. Teubner, Leipzig (2005), p. 42 